\title{
Character Association for Seed Yield and Its Components in Cowpea [Vigna unguiculata (L.)Walp]
}

\author{
P.P. Sharma*, Bhagwati Baranda, Santra Haritwal and Mahesh Sharma
}

Department of Plant Breeding and Genetics, Rajasthan College of Agriculture, Maharana Pratap

University of Agriculture and Technology, Udaipur (Rajasthan)-313001, India

*Corresponding author

\section{A B S T R A C T}

\begin{tabular}{|l|}
\hline Ke y w or d s \\
Cowpea, \\
Correlation, \\
Path analysis. \\
\hline Article Info \\
\hline Accepted: \\
17 July 2017 \\
Available Online: \\
10 September 2017 \\
\hline
\end{tabular}

Thirty genotypes of cowpea were grown during the rainy season of 2014-2015 to study the interrelationship among quantitative traits. The experiment was laid out in a randomized complete block design (RCBD) with three replications at the Research Field of the Department of Plant Breeding and Genetics, MPUAT University, Rajasthan. Correlation studies revealed that seed yield was significantly and positively correlated with clusters per plant $\left(\mathrm{rg}=0.466^{* *}, \mathrm{rp}=0.361^{* *}\right)$, pods per plant $\left(\mathrm{rg}=0.505^{* *}, \mathrm{rp}=0.442^{* *}\right)$, biological yield $\left(0.738^{* *}, \mathrm{rp}=0.705^{* *}\right)$, harvest index $\left(0.223^{*}, \mathrm{rp}=0.354^{* *}\right)$ and 100 -grain weight $\left(0.354^{* *}, \mathrm{rp}=0.340^{* *}\right)$. The path coefficient analysis based on seed yield, as a dependent variable, revealed that biological yield had the greatest positive direct effect on seed yield (1.228) followed by harvest index (0.828), pod length (0.109) and branches per plant(0.107). Correlation and path analysis indicated that for increasing seed yield in cowpea direct selection should be carried out for higher values of biological yield per plant, harvest index, pod length and primary branches per plant.

\section{Introduction}

Cowpea (Vigna unguiculata L. Walp) is one of the important food legumes in the hot-dry tropics and sub- tropics and especially, in the sub-Saharan Africa (Uarrota, 2010). Cowpea plays a substantial role by serving as a grain and vegetable crop mainly for the rural people in the East, West, South and Central parts of Africa (Mortimore et al., 1997).Cowpea, also popularly called 'beans' is mostly grown in dry areas in mixtures. It is thought that the origin of the cultivated species of cowpea is Africa (Gibbon and Pain, 1988). Cowpea is a legume of significant economic importance worldwide. It is an important versatile food crop. It is one of the most ancient crops known to man. The off-take crop of cowpea for fodder makes an important contribution to feed supplies for ruminants to maintain their health in dry season (Quin, 1997). Dry seeds of cowpea are used to prepare several snacks and main meal dishes. Its grain contains high amount of quality protein (23.4\%), carbohydrate $(60.3 \%)$ and fat $(1.8 \%)$. The knowledge of character association i.e. genotypic and phenotypic correlation between yield and its component characters is essential for yield improvement through selection programme (Fraser and Eaton, 1983). Causeeffect analysis provides an effective means of partitioning the correlation coefficients into 
direct and indirect effects of the component characters on yield based on which crop improvement programme can be logically devised (Rao et al., 1997).

\section{Materials and Methods}

Thirty genotypes including three checks (RC101, RC-19 and RCV-7) were evaluated in kharif, 2015 crop was raised. Each crop was sown in randomized block design with three replications keeping two rows of each genotype in a plot of $4 \mathrm{~m}$ length in each replication with the spacing of $30 \times 10 \mathrm{~cm}$. Recommended and uniform agronomical practices was adopted for all the three environments. In each environment, observation were recorded on five randomly selected competitive plants viz., Plant height (cm), Number of branches per plant, Number of pods per plant, Number of clusters per plant, Pod length $(\mathrm{cm})$, Number of seeds per pod, 100-grain weight (g), Seed yield per plant (g), Biological yield per plant (g), Harvest index (\%) and Seed protein content (\%). Whereas, Days to $50 \%$ flowering and Days to $75 \%$ maturity were recorded on plot basis.

Replication wise data for each character were subjected to analysis of variance. The genotypic and phenotypic component of variances and covariance as described by Singh and Choudhary (1995) and as per formula given by Johnson et al., (1955). The direct and indirect effects were calculated according to path analysis as suggested by Wright (1921) and elaborated by Dewey and Lu (1959).

\section{Genotypic covariance $\left(\operatorname{Cov}_{\mathrm{gxy}}\right)$}

The formula for calculating genotypic covariance is described as below:

$\operatorname{Cov}_{\text {gxy }}=(\operatorname{MSPg}-\mathrm{MSPe}) / \mathrm{r}$
Where,

MSPg = Mean sum of products due to genotypes between variable $\mathrm{x}$ and $\mathrm{y}$.

MSPe $=$ Mean sum of products due to error between variable $\mathrm{x}$ and $\mathrm{y}$.

$\mathrm{r}=$ Number of replication

\section{Phenotypic covariance $\left(\operatorname{Cov}_{\mathrm{pxy}}\right)$}

The formula for calculating phenotypic covariance is explained as under:

$\operatorname{Cov}_{\mathrm{pxy}}=\operatorname{Covgxy}+\operatorname{Cov}_{\mathrm{exy}}$

Where,

Error covariance $\left(\operatorname{Cov}_{\text {exy }}\right)=\mathrm{MSPe}$

$\mathrm{r}=$ Number of replication

Genotypic correlation coefficient $\left(\mathbf{r}_{\mathrm{gxy}}\right)$

$r_{x y}(g)=\frac{\operatorname{Cov} \cdot x y(g)}{\sqrt{V x(g) \cdot V y(g)}}$

Where,

Cov (xy) $\mathrm{g}=$ Genotypic covariance between two characters $\mathrm{x}$ and $\mathrm{y}$.

$\sigma_{\mathrm{gx}}^{2}=$ Genotypic variance for character $\mathrm{x}$.

$\sigma_{\text {gy }}^{2}=$ Genotypic variance for character $\mathrm{y}$.

Phenotypic correlation coefficient

$r_{x y}(p h)=\frac{\operatorname{Cov} \cdot x y(p h)}{\sqrt{V x(p h) \cdot V y(p h)}}$

Where, 
Cov $(x y) p=$ Phenotypic covariance between two character $\mathrm{x}$ and $\mathrm{y}$.

$\sigma_{p x}^{2}=$ Phenotypic variance for character $\mathrm{x}$.

$\sigma_{\text {py }}^{2}=$ Phenotypic variance for character $y$.

Path coefficients were analyzed at genotypic level for seed yield per plant. The direct and indirect effects of 12 characters on seed yield per plant (Y) were obtained as per procedure given below.

$\mathrm{r}_{1 \mathrm{Y}} \mathrm{r}_{1(1)} \mathrm{r}_{1(2)} \quad \mathrm{r}_{1(12)} \mathrm{P}_{1(\mathrm{Y})}$

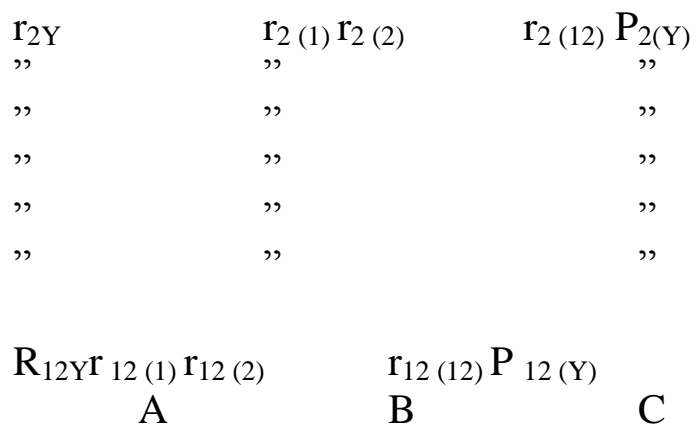

Where,

$\mathrm{r}_{1 \mathrm{Y}}, \mathrm{r}_{2 \mathrm{Y}}, \mathrm{r}_{3 \mathrm{Y}}, \ldots \ldots \ldots \ldots . . \mathrm{r}_{12 \mathrm{Y}}$ are the genotypic correlation of, days to $50 \%$ flowering, Days to $75 \%$ maturity, Plant height $(\mathrm{cm})$, Number of branches per plant, Number of pods per plant, Number of clusters per plant, Pod length (cm), Number of seeds per pod, 100-grain weight (g), Biological yield per plant (g),Harvest index (\%), Seed protein content (\%), respectively.

$\mathrm{P}_{1 \mathrm{Y}}, \mathrm{P}_{2 \mathrm{Y}}, \mathrm{P}_{3 \mathrm{Y}}, \ldots \ldots \ldots \ldots \ldots \ldots \mathrm{P}_{13 \mathrm{Y}}$ effects of days to $50 \%$ flowering, Days $75 \%$ to maturity, Plant height $(\mathrm{cm})$,Number of branches per plant, Number of pods per plant, Number of clusters per plant, Pod length (cm), Number of seeds per pod,100-grain weight $(\mathrm{g})$, Biological yield per plant $(\mathrm{g})$, Harvest index (\%), Seed protein content $(\%)$, respectively.
Or

$\mathrm{A}=\mathrm{BC}$

Values of ' $\mathrm{C}$ ' vector were obtained as:

$\mathrm{C}=\mathrm{B}^{-1} \mathrm{~A}$

Where,

$\mathrm{A}$ is the vector $\mathrm{f}$ direct correlations of fifteen characters with yield $\mathrm{Y}$.

$\mathrm{B}^{-1}$ is the inverse of mutual correlation matrix of characters.

$\mathrm{C}$ is the vector of direct effects.

The inverse of this matrix was carried out by Pivotal Condensation Method (Singh and Chaudhary, 1979).

The residual effect was computed from the following algebraic relationship:

$$
\begin{aligned}
& 1=R^{2}+r_{1} Y_{1} Y+r_{2} Y P_{2} Y+r_{3} Y P_{3} Y+r_{4} \\
& Y P_{4} Y+r_{5} P_{5} Y+r_{6} Y P_{6} Y+r_{7} \mathrm{YP}_{7} Y+r_{8} \\
& Y P_{8} Y+r_{9} Y P_{9} Y+r_{10} Y P_{10} Y+r_{11} Y_{11} Y
\end{aligned}
$$

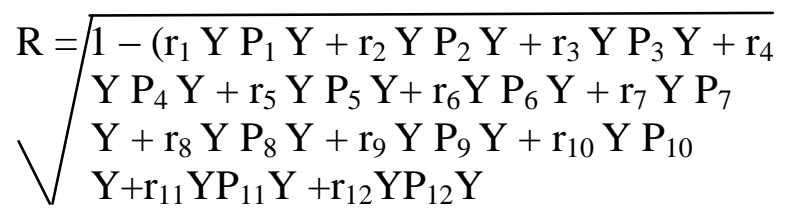

Where, $\mathrm{R}$ is the residual effect.

\section{Results and Discussion}

Phenotypic correlation is directly proportional to genotypic and environmental correlations. On the other hand, a positive genetic correlation between two desirable traits makes selection easy for improving both the traits simultaneously while the reverse is the case for negative correlation. In the present study, genotypic correlation coefficients were higher 
than corresponding phenotypic correlation coefficients, indicating greater contribution of genotypic factor in expression of these traits association.

Correlation studies revealed that seed yield was exhibited significantly and positively correlated with clusters per plant ( $\mathrm{rg}=$ $\left.0.466^{* *}, \mathrm{rp}=0.361^{* *}\right)$, pods per plant $(\mathrm{rg}=$ $\left.0.505^{* *}, \quad \mathrm{rp}=0.442 * *\right)$, biological yield $\left(0.738 * *, \mathrm{rp}=0.705^{* *}\right)$, harvest index $\left(0.223^{*}\right.$, $\mathrm{rp}=0.354 * *)$ and 100 -grain weight $(0.354 * *$, $\left.\mathrm{rp}=0.340^{* *}\right)$ at both genotypic and phenotypic level. Sharma et al., (2016)observed that seed yield per plant showed significant positive correlation with number of cluster per plant, number of pods per plant, biological yield per plant, harvest index and 100-grain weight.

These finding also supported by Sharma et al., (2016), Bhardwaj et al., (2014) and Bhradu and Navale (2011).

Whereas, seed yield showed significant genotypic correlation in negative direction with number of branches per plant $(\mathrm{rg}=$ $\left.0.215^{*}\right)$. The present findings are in accordance with the findings of Padi et al., (2003), Fana et al., (2004) and Kaveris et al., (2007).

Seed protein content showed significantly positive correlation with pod length $\left(\mathrm{rg}=0.223^{*}\right)$ at only genotypically. Whereas, 100-grain weight exhibited significant association in positive direction at both genotypically and phenotypically with days to $75 \%$ maturity $(\mathrm{rg}=0.334 * *, \mathrm{rp}=0.309 * *)$, number of branches per plant $\left(\mathrm{rg}=0.275^{* *}\right.$, $\left.\mathrm{rp}=0.271^{* *}\right)$, pod length $\left(\mathrm{rg}=0.575^{* *}, \mathrm{rp}=\right.$ $\left.0.524^{* *}\right)$ and biological yield per plant $(\mathrm{rg}=$ $0.540 * *, \quad \mathrm{rp}=0.540 * *)$ while, significant negative correlation with harvest index ( $\mathrm{rg}=$ $0.287 * *, \quad \mathrm{rp}=-0.270 * *$ ) also reported by Sharma et al., (2016) revealed that 100-grain weight exhibit significant positive correlation with number of branches per plant and harvest index and Selvakumar and Ushakumari (2013) for pod length and days to maturity.

Table 1 perusal that harvest index revealed significant and genotypic correlation in positive direction $(\mathrm{rg}=0.391 * *)$ as well as significant negative phenotypic correlation with days to $50 \%$ flowering $\left(\mathrm{rp}=-0.239^{*}\right)$. However, harvest index showed significant negative correlation at both genotypically and phenotypically with number of branches per plant $\left(\mathrm{rg}=-0.302 * * \& \mathrm{rp}=-0.266^{*}\right)$, biological yield per plant $(\mathrm{rg}=-0.485 * * \& \mathrm{rp}=$ $-0.448 * *)$ and days to $75 \%$ maturity ( $\mathrm{rg}=-$ $0.549 * * \& \mathrm{rp}=-0.482 * *)$.

Sharma et al., (2016) revealed that harvest index exhibit significant negative correlation with days to maturity and biological yield at both genotypic and phenotypic level.

Biological yield per plant exhibited significant positive correlation at both genotypic and phenotypic level with days to $75 \%$ maturity ( $\mathrm{rg}=0.502 * * \& \mathrm{rp}=0.450 * *)$, number of pods per plant $(\mathrm{rg}=0.439 * * \&$ $\left.\mathrm{rp}=0.380^{* *}\right)$, number of clusters per plant $\left(\mathrm{rg}=0.411^{* *} \& \mathrm{rp}=0.326^{* *}\right)$, days to $50 \%$ flowering ( $\left.\mathrm{rg}=0.375^{* *} \& \mathrm{rp}=0.235^{*}\right)$ and pod length $\left(\mathrm{rg}=0.248^{*} \& \mathrm{rp}=0.231^{*}\right)$. Similar result also reported by Sharma et al., (2016) and Manggoel et al., (2012).

Number of seeds per pod showed significant positive correlation at both genotypic and phenotypic level with pod length $(\mathrm{rg}=0.363 * *$ $\& \mathrm{rp}=0.360$ ).

While, significant correlation in negative direction with days to $50 \%$ flowering ( $\mathrm{rg}=$ 0.314) also reported by Sharma et al., (2016), Diriba Shanko et al., (2014) and Selvakumar and Ushakumari (2013) revealed number of seeds per pod showed significant positive correlation with pod length. 
Table.1 Genotypic and phenotypic correlation coefficient between seed yield per plant and other characters in cowpea in E1

\begin{tabular}{|c|c|c|c|c|c|c|c|c|c|c|c|c|c|c|}
\hline Character & $\begin{array}{l}\mathbf{D} / \\
\mathbf{F}\end{array}$ & $\begin{array}{l}\text { Days to } \\
\mathbf{5 0 \%} \\
\text { Flowering }\end{array}$ & $\begin{array}{l}\text { Days to } \\
\mathbf{7 5 \%} \\
\text { Maturity }\end{array}$ & $\begin{array}{r}\text { Plant } \\
\text { Height } \\
(\mathrm{cm}) \\
\end{array}$ & $\begin{array}{l}\text { Branches } \\
\text { / Plant }\end{array}$ & $\begin{array}{l}\text { Cluster/ } \\
\text { Plant } \\
\end{array}$ & $\begin{array}{l}\text { Pods/ } \\
\text { Plant } \\
\end{array}$ & $\begin{array}{l}\text { Pod } \\
\text { Length } \\
\text { (cm) }\end{array}$ & Seed/Pod & $\begin{array}{l}\text { Biological } \\
\text { Yield/ } \\
\text { Plant (g) }\end{array}$ & $\begin{array}{l}\text { Harvest } \\
\text { Index } \\
(\%)\end{array}$ & $\begin{array}{l}100- \\
\text { grain } \\
\text { Weight } \\
\text { (g) } \\
\end{array}$ & $\begin{array}{l}\text { Seed } \\
\text { Protein } \\
\text { Content } \\
(\%) \\
\end{array}$ & $\begin{array}{l}\text { Seed } \\
\text { Yield/ } \\
\text { Plant (g) }\end{array}$ \\
\hline \multirow[t]{2}{*}{ Days to $50 \%$ Flowering } & G & 1.000 & $0.786^{* *}$ & $0.264 *$ & 0.006 & $0.232 *$ & $0.232 *$ & -0.280 ** & $-0.314 * *$ & $0.375^{* *}$ & $0.391 * *$ & 0.144 & -0.134 & 0.140 \\
\hline & $\mathrm{P}$ & 1.000 & $0.514 * *$ & 0.192 & 0.007 & 0.156 & 0.102 & -0.197 & -0.132 & $0.235^{* *}$ & $-0.239^{*}$ & 0.104 & -0.097 & 0.143 \\
\hline \multirow[t]{2}{*}{ Days to $75 \%$ Maturity } & $\mathrm{G}$ & & 1.000 & $0.219 *$ & $0.290^{* * *}$ & -0.034 & -0.060 & -0.157 & -0.177 & $0.502 * *$ & $-0.549 * *$ & $0.334 * *$ & -0.141 & 0.167 \\
\hline & $\mathrm{P}$ & & 1.000 & 0.201 & $0.274 * *$ & 0.014 & -0.052 & -0.133 & -0.171 & $0.450^{* *}$ & $-0.482 * *$ & $0.309 * *$ & -0.133 & 0.142 \\
\hline \multirow[t]{2}{*}{ Plant Height $(\mathrm{cm})$} & G & & & 1.000 & 0.032 & 0.074 & 0.148 & $-0.314 * *$ & 0.201 & 0.176 & -0.063 & -0.168 & 0.010 & 0.193 \\
\hline & $\mathrm{P}$ & & & 1.000 & 0.030 & 0.065 & 0.131 & $-0.287 * *$ & 0.191 & 0.173 & -0.060 & -0.168 & 0.010 & 0.188 \\
\hline \multirow[t]{2}{*}{ Branches/ Plant } & G & & & & 1.000 & $0.471^{* *}$ & $-0.471 * *$ & 0.179 & 0.068 & -0.046 & $-0.302 * *$ & $0.275^{* *}$ & 0.022 & $-0.215^{*}$ \\
\hline & $\mathrm{P}$ & & & & 1.000 & $-0.412 * *$ & $-0.385^{* *}$ & 0.149 & 0.062 & -0.036 & $-0.266^{*}$ & $0.271 * *$ & 0.022 & -0.198 \\
\hline \multirow[t]{2}{*}{ Cluster/ Plant } & $\mathrm{G}$ & & & & & 1.000 & $0.869^{* * *}$ & -0.080 & 0.036 & $0.411^{* *}$ & 0.020 & -0.098 & -0.155 & 0.466 ** \\
\hline & $\mathrm{P}$ & & & & & 1.000 & $0.638^{* *}$ & -0.044 & 0.016 & $0.326^{* *}$ & -0.037 & -0.082 & -0.131 & $0.361 * *$ \\
\hline \multirow[t]{2}{*}{ Pods/ Plant } & $\mathrm{G}$ & & & & & & 1.000 & -0.056 & -0.063 & $0.439 * *$ & 0.043 & -0.157 & 0.056 & $0.505 * *$ \\
\hline & $\mathrm{P}$ & & & & & & 1.000 & -0.067 & -0.064 & $0.380^{* * *}$ & 0.021 & -0.136 & 0.048 & $0.442 * *$ \\
\hline \multirow[t]{2}{*}{ Pod Length $(\mathrm{cm})$} & $\mathrm{G}$ & & & & & & & 1.000 & $0.363^{* *}$ & $0.248^{*}$ & -0.095 & $0.575^{* *}$ & $0.223 *$ & 0.191 \\
\hline & $\mathrm{P}$ & & & & & & & 1.000 & $0.360^{* * *}$ & $0.231^{*}$ & -0.093 & $0.524 * *$ & 0.204 & 0.169 \\
\hline \multirow[t]{2}{*}{ Seed/ Pod } & $\mathrm{G}$ & & & & & & & & 1.000 & -0.010 & 0.040 & 0.052 & 0.002 & 0.011 \\
\hline & $\mathrm{P}$ & & & & & & & & 1.000 & -0.013 & 0.029 & 0.046 & 0.002 & 0.024 \\
\hline \multirow[t]{2}{*}{$\begin{array}{l}\text { Biological Yield/ Plant } \\
(\mathrm{g})\end{array}$} & $\mathrm{G}$ & & & & & & & & & 1.000 & $-0.485 * *$ & $0.558 * *$ & -0.063 & $\mathbf{0 . 7 3 8} * *$ \\
\hline & $\mathrm{P}$ & & & & & & & & & 1.000 & $-0.448 * *$ & $0.540 * *$ & -0.064 & $0.705^{* *}$ \\
\hline \multirow[t]{2}{*}{ Harvest Index (\%) } & $\mathrm{G}$ & & & & & & & & & & 1.000 & $-0.287 * *$ & -0.080 & $0.223 * *$ \\
\hline & $\mathrm{P}$ & & & & & & & & & & 1.000 & $-0.270 * *$ & -0.073 & $0.244 * *$ \\
\hline \multirow[t]{2}{*}{ 100-grain weight (g) } & $\mathrm{G}$ & & & & & & & & & & & 1.000 & -0.106 & $0.354 * *$ \\
\hline & $\mathrm{P}$ & & & & & & & & & & & 1.000 & -0.106 & $0.340 * *$ \\
\hline \multirow[t]{2}{*}{$\begin{array}{l}\text { Seed Protein Content } \\
(\%)\end{array}$} & $\mathrm{G}$ & & & & & & & & & & & & 1.000 & -0.146 \\
\hline & $\mathrm{P}$ & & & & & & & & & & & & 1.000 & -0.143 \\
\hline
\end{tabular}

*and $* *$ significance levels of $5 \%$ and $1 \%$ respectively 
Table. 2 Genotypic and phenotypic path matrix for seed yield in cowpea in E1

\begin{tabular}{|c|c|c|c|c|c|c|c|c|c|c|c|c|c|c|}
\hline Character & $\begin{array}{l}\mathrm{D} \\
/ \mathrm{F}\end{array}$ & $\begin{array}{l}\text { Days to } \\
\mathbf{5 0 \%} \\
\text { Flowering } \\
\end{array}$ & $\begin{array}{l}\text { Days to } \\
\mathbf{7 5 \%} \\
\text { Maturity } \\
\end{array}$ & $\begin{array}{l}\text { Plant } \\
\text { Height } \\
\text { (cm) }\end{array}$ & $\begin{array}{l}\text { Branche } \\
\text { s/ Plant }\end{array}$ & $\begin{array}{l}\text { Cluster/ } \\
\text { Plant } \\
\end{array}$ & $\begin{array}{l}\text { Pods/ } \\
\text { Plant }\end{array}$ & $\begin{array}{l}\text { Pod } \\
\text { Length } \\
\text { (cm) }\end{array}$ & $\begin{array}{l}\text { Seed/ } \\
\text { Pod }\end{array}$ & $\begin{array}{l}\text { Biological } \\
\text { Yield/ } \\
\text { Plant (g) } \\
\end{array}$ & $\begin{array}{l}\text { Harvest } \\
\text { Index }(\%)\end{array}$ & $\begin{array}{l}\text { 100-grain } \\
\text { Weight } \\
\text { (g) }\end{array}$ & $\begin{array}{l}\text { Seed } \\
\text { Protein } \\
\text { Content } \\
(\%) \\
\end{array}$ & $\begin{array}{l}\text { Seed Yield/ } \\
\text { Plant }(\mathrm{g})\end{array}$ \\
\hline \multirow[t]{2}{*}{ Days to $50 \%$ Flowering } & $\mathrm{G}$ & 0.047 & 0.037 & 0.012 & 0.000 & 0.011 & 0.011 & -0.013 & -0.015 & 0.017 & -0.018 & 0.007 & -0.006 & 0.140 \\
\hline & $\mathrm{P}$ & $\mathbf{0 . 0 7 7}$ & 0.040 & 0.015 & 0.001 & 0.012 & 0.008 & -0.015 & -0.010 & 0.018 & -0.019 & 0.008 & -0.008 & 0.143 \\
\hline \multirow[t]{2}{*}{ Days to $75 \%$ Maturity } & G & -0.006 & -0.007 & -0.002 & -0.002 & 0.000 & 0.000 & 0.001 & 0.001 & -0.004 & 0.004 & -0.002 & 0.001 & 0.167 \\
\hline & $\mathrm{P}$ & -0.004 & -0.008 & -0.002 & -0.002 & 0.000 & 0.000 & 0.001 & 0.001 & -0.003 & 0.004 & -0.002 & 0.001 & 0.142 \\
\hline \multirow[t]{2}{*}{ Plant Height $(\mathrm{cm})$} & $\mathrm{G}$ & 0.011 & 0.009 & 0.043 & 0.001 & 0.003 & 0.006 & -0.013 & 0.009 & 0.008 & -0.003 & -0.007 & 0.000 & 0.193 \\
\hline & $\mathrm{P}$ & 0.011 & 0.011 & 0.055 & 0.002 & 0.004 & 0.007 & -0.016 & 0.011 & 0.010 & -0.003 & -0.009 & 0.001 & 0.188 \\
\hline \multirow[t]{2}{*}{ Branches/ Plant } & $\mathrm{G}$ & 0.001 & 0.031 & 0.003 & $\mathbf{0 . 1 0 7}$ & -0.050 & -0.050 & 0.019 & 0.007 & -0.005 & -0.032 & 0.029 & 0.002 & $-0.215 *$ \\
\hline & $\mathrm{P}$ & 0.000 & 0.017 & 0.002 & 0.063 & -0.026 & -0.024 & 0.009 & 0.004 & -0.002 & -0.017 & 0.017 & 0.001 & -0.198 \\
\hline \multirow[t]{2}{*}{ Cluster/ Plant } & $\mathrm{G}$ & 0.024 & -0.004 & 0.008 & -0.048 & 0.103 & 0.089 & -0.008 & 0.004 & 0.042 & 0.002 & -0.010 & -0.016 & $0.466 * *$ \\
\hline & $\mathrm{P}$ & 0.009 & 0.001 & 0.004 & -0.023 & 0.055 & 0.035 & -0.002 & 0.001 & 0.018 & -0.002 & -0.005 & -0.007 & $0.361 * *$ \\
\hline \multirow[t]{2}{*}{ Pods/ Plant } & G & -0.036 & 0.009 & -0.023 & 0.072 & -0.133 & -0.153 & 0.009 & 0.010 & -0.067 & -0.007 & 0.024 & -0.009 & $0.505 * *$ \\
\hline & $\mathrm{P}$ & 0.003 & -0.002 & 0.004 & -0.012 & 0.020 & $\mathbf{0 . 0 3 2}$ & -0.002 & -0.002 & 0.012 & 0.001 & -0.004 & 0.002 & $0.442 * *$ \\
\hline \multirow[t]{2}{*}{ Pod Length $(\mathrm{cm})$} & $\mathrm{G}$ & -0.030 & -0.017 & -0.034 & 0.019 & -0.009 & -0.006 & 0.109 & 0.040 & 0.027 & -0.010 & 0.063 & 0.024 & 0.191 \\
\hline & $\mathrm{P}$ & -0.013 & -0.009 & -0.019 & 0.010 & -0.003 & -0.004 & 0.066 & 0.024 & 0.015 & -0.006 & 0.035 & 0.013 & 0.169 \\
\hline \multirow[t]{2}{*}{ Seed/ Pod } & G & 0.017 & 0.010 & -0.011 & -0.004 & -0.002 & 0.004 & -0.020 & -0.055 & 0.001 & -0.002 & -0.003 & 0.000 & 0.011 \\
\hline & $\mathrm{P}$ & 0.001 & 0.002 & -0.002 & -0.001 & 0.000 & 0.001 & -0.004 & -0.011 & 0.000 & 0.000 & -0.001 & 0.000 & 0.024 \\
\hline \multirow[t]{2}{*}{$\begin{array}{l}\text { Biological Yield/ Plant } \\
(\mathrm{g})\end{array}$} & $\mathrm{G}$ & 0.461 & 0.616 & 0.216 & -0.057 & 0.504 & 0.539 & 0.305 & -0.012 & 1.228 & -0.596 & 0.685 & -0.078 & $\mathbf{0 . 7 3 8} * *$ \\
\hline & $\mathrm{P}$ & 0.229 & 0.438 & 0.168 & -0.035 & 0.317 & 0.370 & 0.225 & -0.012 & $\mathbf{0 . 9 7 3}$ & -0.436 & 0.526 & -0.062 & $0.705 * *$ \\
\hline \multirow[t]{2}{*}{ Harvest Index (\%) } & $\mathrm{G}$ & -0.323 & -0.454 & -0.052 & -0.250 & 0.017 & 0.036 & -0.079 & 0.033 & -0.402 & 0.828 & -0.238 & -0.066 & $0.223 *$ \\
\hline & $\mathrm{P}$ & -0.170 & -0.342 & -0.043 & -0.189 & -0.026 & 0.015 & -0.066 & 0.020 & -0.318 & 0.711 & -0.192 & -0.052 & $0.244 *$ \\
\hline \multirow[t]{2}{*}{ 100-grain Weight $(\mathrm{g})$} & G & -0.028 & -0.066 & 0.033 & -0.054 & 0.019 & 0.031 & -0.113 & -0.010 & -0.109 & 0.056 & -0.196 & 0.021 & $\mathbf{0 . 3 5 4} * *$ \\
\hline & $\mathrm{P}$ & -0.004 & -0.011 & 0.006 & -0.010 & 0.003 & 0.005 & -0.019 & -0.002 & -0.020 & 0.010 & -0.036 & 0.004 & $0.340 * *$ \\
\hline \multirow[t]{2}{*}{ Seed Protein Content $(\%)$} & $\mathrm{G}$ & 0.003 & 0.003 & 0.000 & 0.000 & 0.003 & -0.001 & -0.004 & 0.000 & 0.001 & 0.002 & 0.002 & -0.020 & -0.146 \\
\hline & $\mathrm{P}$ & 0.003 & 0.005 & 0.000 & -0.001 & 0.005 & -0.002 & -0.007 & 0.000 & 0.002 & 0.003 & 0.004 & -0.035 & -0.143 \\
\hline
\end{tabular}

(R square $=1.0057$ and Residual effect $=0.07549)($ Phenotypic $\mathrm{R} 2=0.9060$ and Residual effect $=0.3067)$ 
Pod length showed significant correlation in negative direction with plant height ( $\mathrm{rg}=$ $0.314 * * \& \mathrm{rp}=-0.287$ )at both genotypic and phenotypic level as well significant negative correlation with days to $50 \%$ flowering ( $\mathrm{rg}=$ $0.280^{* *}$ ) at genotypic level also reported by Sharma et al., (2016) revealed that pod length exhibit significant negative correlation with days to $50 \%$ flowering. Similar studies was done by Diriba Shanko et al., (2014) and Selvakumar and Ushakumari (2013).

Table 1 further revealed that number of pods per plant exhibited significant positive correlation with number of clusters per plant $\left(\mathrm{rg}=0.869^{* *}, \mathrm{rp}=0.638^{* *}\right)$ at both genotypic and phenotypic level and with days to $50 \%$ flowering $(\mathrm{rg}=0.232 *)$ at only genotypic level, while number of pods per plant exhibited significant negative correlation with branches per plant $(\mathrm{rg}=-0.471 * * \& \mathrm{rp}=-$ $\left.0.385^{* *}\right)$.

Number of clusters per plant exhibited significant association in positive direction with number of branches per plant $\left(0.471^{* *}\right)$ and days to $50 \%$ flowering $(\mathrm{rg}=0.232 *)$ both at genotypic level, while significant negative correlation with number of branches per plant $(\mathrm{rp}=-0.412 * *)$ at only phenotypic level. Similar finding was also reported by Sharma et al., (2016) and Selvakumar and Ushakumari (2013).

Number of branches per plant showed significant association in positive direction with days to $75 \%$ maturity $(\mathrm{rg}=0.290 * * \& \mathrm{rp}=$ $0.274 * *)$ at both genotypic and phenotypic level in $\mathrm{E}_{1}$ and days to $50 \%$ flowering $\left(\mathrm{rg}=0.335^{* *}\right)$ also showed significant positive genotypic correlation over pooled basis. Similar studies also done by Sharma et al., (2016) and Kumari et al., (2010) revealed that number of primary branches per plant exhibited significant positive correlation with day to maturity.
Plant height showed significantly positive correlation at genotypic level with days to $50 \%$ flowering $(\mathrm{rg}=0.264 *)$ and days to $75 \%$ maturity $(\mathrm{rg}=0.219 *)$. Further days to $75 \%$ maturity showed significant positive correlation at both genotypically and phenotypically with days to $50 \%$ flowering $(\mathrm{rg}=0.786 * * \& \mathrm{rp}=0.514 * *)$.

Significant and positive genotypic correlation coefficients are an indication that selection of genotypes for future breeding programme should be fundamentally based on the superiority of the genotypes alongside with their phenotypic expression. This implies that seeds per pod, primary branch per plant and pod length should be given high priority during selection in cowpea. Similar finding are also reported by Venkatesen et al., (2003), Patil et al., (2004) and Manggoel et al., (2012).

The direct and indirect effects of different characters on seed yield per plant were worked out using path coefficient analysis. Among the different contributing characters high positive direct effects on seed yield per plant were exhibited by biological yield (1.228), harvest index (0.828), pod length (0.109), number of branches per plant(0.107) and clusters per plant(0.55) at genotypic level (Table 2) and positive direct effects on seed yield per plant were exhibited by biological yield (0.973), harvest index (0.711), days to $50 \%$ flowering (0.077), pod length (0.066) and branches per plant $(0.063)$ at phenotypic level (Table 2) also similar result reported by Madhavi et al., (2014), Kwago (2014) and Diribashanko et al., (2014).

The highest positive indirect effect through 100 -grain weight by biological yield per plant (0.685) followed by days to $75 \%$ maturity (0.616), pods per plant (0.539), clusters per plant (0.504) and days to $50 \%$ flowering (0.461) at genotypic level and the highest 
positive indirect effect through 100-grain weight at phenotypic level by biological yield (0.973) followed by harvest index (0.711), days to $50 \%$ flowering (0.077), pod length (0.066), branches per plant (0.063) and clusters per plant (0.055).

Seed yield per plant had significant positive correlation with clusters per plant, pods per plant, biological yield, harvest index and 100grain weight. Among these traits seeds per pod, primary branches per plant, biological yield, harvest index, pods per plant and pods length had very high correlation and were also showing high positive indirect effects on seed yield per plant. Hence, these traits can be used for effective selection as well as breeding of superior genotypes for enhancing seed yield in cowpea. Residual effect in path analysis was found to be 0.075 for genotypic level and 0.306 for phenotypic level showing that there is maximum effect of the traits studied on seed yield in cowpea.

The present study illustrated the phenotypic and genotypic correlations showing positive correlation of seed yield with important agromorphological characters. Hence, improving one or more of these traits could result in high seed yield for cowpea. Clusters per plant, pods per plant, biological yield, harvest index and 100-grain weight had high positive genotypic direct effects and correlation with seed yield per plant suggesting the possibility of improving seed yield through direct selection of these traits.

\section{References}

Bhardu, D., and Navale, P.A. 2011. Correlation and path analysis studies in $\mathrm{F}_{3}$ population of cowpea [Vigna unguiculata (L.) Walp]. Legume Res, 34:41-44.

Bhardwaj, R., Singh, D.P., Rani, U., Tiwana, U.S. and Bajaj, R.K. 2014. Association studies in cowpea [Vigna unguiculata (L.) Walp]. Journal of Food Legumes, 27(1): 74-76.

Fana, S.B., Pasquet, R.S. and Gepts, P. 2004. Genetic diversity in cowpea [Vigna unguiculata (L.) Walp] as revealed by RAPD markers.Genetic Resources and Crop Evaluation, 51:539-550.

Fraser, J., and Eaton, G.W. 1983. Application of yield component analysis to crop research. Field Crop Abstracts, 36: 787797.

Gibbon, D. and Pain, A. 1988. Crops of the drier regions of the tropics. ELBS / Longman.157 pp.

Johnson, H.W., Robinson, H.F. and Comstock, R.F. 1955. Estimation of genetic environmental variability in soybean. Agronomy Journal, 47: 314318.

Kaveries, B., Salimath, P.M. and Ravikumar, R.L. 2007. Genetic Studies in Greengram and association analysis. Karnataka Journal of Agricultural Science, 20:843-844.

Kumari, R. U., Usharani, K S., Suguna, R. and Anandakumar, C.R. 2010. Relationship between the yields contributing character in cowpea for grain purpose [Vigna unguiculata (L.) Walp]. Electronic Journal of Plant Breeding, 1(4): 882-884.

Kwaga, Y.M., 2014. Direct and indirect contribution of yield attributes to the grain yield of cowpea [Vigna unguiculata (L.) Walp] grown in Northern Guinea Savanna, Nigeria. Research Journal of Pharmaceutical, Biological and Chemical Sciences, 5(2): pg- 1671.

Madhavi, K., Sivaji, T. and Amrutha, N. 2014. A Path coefficient analysis for growth, yield and yield components in vegetable cowpea. [Vigna unguiculata (L.)Walp]. National Academy of Agriculture Science, 32: 1-2. 
Manggoel, W., Uguru, M.I., Ndam, O.N. and Dasbak, M.A. 2012. Genetic variability, correlation and path coefficient analysis of some yield components of ten cowpea [Vigna unguiculata (L.) Walp] accessions. Journal of Plant Breeding and Crop Science, 4(5): 80-86.

Meena, H.K., Ram Krishna, K. and Sing, B. 2015. Character associations between seed yield and its components traits in cowpea [Vigna unguiculata (L.)Walp.]. Indian J. Agric. Res., 49(6):S 567-570.

Mortimore, M.J., Singh BB, Harris F, Blade SF 1997. Cowpea in Traditional Cropping Systems. In: Advances in Cowpea Research, Singh, B.B., D.R. Mohan Raj, K.E. Dashiell and L.E.N. Jackai (Eds.). IITA and JIRCAS, Hong Kong, pp 99-113

Padi, F.K., 2003. Correlation and path analysis of yield and yield components pigeon pea. Pakistan Journal of Biological science, 6: 1689-1694.

Quin, F.M., 1997. Introduction. In: Advances in cowpea research. Singh, B.B., Ral, Mohan, Deshiell, K. and Jackai, L.E.N. (Eds.). IITA/JIRCAS, IITA, Ibadan, Nigeria, IX-XV.

Rao, A., Khan, M.A., McNeilly, T. and Khan, A.A. 1997. Cause and effect relations of yield and yield components in rice (Oryza sativa L.). J. Genet. Breed, 51: 1-5.

Sapar, G.K., and Javia, R.M. 2014. Correlation and path analysis in vegetable cowpea [Vigna unguiculata
(L.)Walp]. International Journal of Plant Science, 9: 138-141.

Selvakumar, G., and Ushakumari, R. 2013. Association analysis in the intersubspecific crosses of cowpea [Vigna unguiculata (L.) Walp] and long yard bean [Vigna unguiculata (L.) Walp. spp. Sesquipedalis]. Electronic Journal of Plant Breeding, 4(4): 13361339.

Shanko, D., Andragie, M. and Zelleke, H. 2014. Interrelationship and path coefficient analysis of some growth and yield characteristics in cowpea [Vigna unguiculata (L.) Walp] genotype. Journal of Plant Science, 2(2):97-101.

Sharma, M., Sharma, P.P., Upadhyay, B. and Bairwa, H.L 2016. Study of correlation coefficient and path analysis in cowpea [Vigna unguiculata (L.)Walp] germplasm line. International Journal of Development Research, 6(8): 90119016.

Singh, R.K., and Choudhary, B.D. 1979. Biometrical Methods in Quantitative Genetic Analysis. Kalyani Publishers, Ludhiana-New Delhi, pp.80-100.

Uarrota, V.G., 2010. Response of cowpea (Vigna unguiculata [L.]Walp) to water stress and phosphorus fertilization. $J$. Agron., 9: 87-91.

Venkatesan, M., Prakash, M. and Ganesan, J. 2003. Correlation and path analysis in cowpea [Vigna unguiculata (L.)Walp] Legume Res., 26:105-108.

\section{How to cite this article:}

Sharma, P.P., Bhagwati Baranda, Santra Haritwal and Mahesh Sharma. 2017. Character Association for Seed Yield and Its Components in Cowpea [Vigna unguiculata (L.) Walp]. Int.J.Curr.Microbiol.App.Sci. 6(9): 967-975. doi: https://doi.org/10.20546/ijcmas.2017.609.117 